\title{
Curcumin-loaded Chitosan Tripolyphosphate Nanoparticles as a safe, natural and effective antibiotic inhibits the infection of Staphylococcus aureus and Pseudomonas aeruginosa in vivo
}

\author{
Mirza Ali Mofazzal Jahromi 1, Sharafaldin Al-Musawi 2, Majid Pirestani 3, Mahdi Fasihi Ramandi 1, \\ Kazem Ahmadi 4, Hajar Rajayi 5, Zuhair Mohammad Hassan 5, Mahdi Kamali ${ }^{6}$, Reza Mirnejad 1,* \\ ${ }^{1}$ Molecular Biology Research Center, Baqiyatallah University of Medical Sciences, Tehran, I.R. IRAN \\ 2Departments of Nanobiotechnology, Faculty of Biological Sciences, Tarbiat Modares University, Tehran, I.R. IRAN \\ ${ }^{3}$ Department of Parasitology, Faculty of Medical Sciences, Tarbiat Modares University, Tehran, I.R. IRAN \\ ${ }^{4}$ Department of Immunology and Research Centre of Molecular Biology, Baqiyatallah University of Medical Sciences, \\ Tehran, I.R. IRAN \\ 5Department of Immunology, Faculty of Medical Sciences, Tarbiat Modares University, Tehran, I.R. IRAN \\ ${ }^{6}$ Nanobiotechnology Research Center, Baqiyatallah University of Medical Sciences, Tehran, I.R. IRAN
}

*Corresponding author: Reza Mirnejad, Molecular Biology Research Center, Baqiyatallah University of Medical Sciences, P.O. Box 19398-5487, Tehran, I.R. IRAN. Tel: +98-2182482554, Fax:+98-2188039883, E-mail: rmirnejad@yahoo.com

Received: February 23, 2014; Revised: May 06, 2014

\begin{abstract}
Background: Curcumin as a yellow natural compound extracted from turmeric root is known it as an antibacterial agent. One of the nanoparticles ability is to decrease the defects of usual drug delivery systems. Chitosan is a low toxic, biodegradable, biocompatible and safe polymer which is used in production of nanoparticles. Nanoparticles like chitosan-tripolyphosphate (TPP) are able to increase antibacterial properties of curcumin.

Materials and Methods: Curcumin-loaded chitosan-TPP nanoparticles containing chitosan, curcumin and TPP salt were synthesized by ionotropic gelation methods. First, the skin of anesthetized mice was inoculated with staphylococcus aureus and pseudomonas aeruginosa suspension. Then the infected mice were treated with curcumin-loaded chitosan-TPP nanoparticles for 3 days. Following that, antibacterial characteristics of the mice treated with curcumin-loaded chitosanTPP nanoparticles were evaluated by bacterial culture of these mice.

Results: Our results showed the size of $160 \pm 10 \mathrm{~nm}$ and the charge of $+7 \pm 2 \mathrm{mV}$ in curcumin-loaded chitosan-TPP nanoparticles. These nanoparticles were also spiral shape. The encapsulation efficiency of curcumin in chitosan-TPP nanoparticles was $75 \pm 2 \%$. Bacterial culture showed that curcumin-loaded chitosan-TPP nanoparticles inhibited staphylococcus aureus and pseudomonas aeruginosa growth.

Conclusions: Our study demonstrated that curcumin-loaded chitosan-TPP nanoparticles can be utilized as a potent agent in treatment of Staphylococcus aureus and Pseudomonas aeruginosa infections.

Keywords: Chitosan-TPP; Curcumin; Nanoparticles; Pseudomonas aeruginosa; Staphylococcus aureus
\end{abstract}

\section{Background}

Curcumin as a natural yellow pigment and a nontoxic, bioactive agent of turmeric separated from the root of Curcumin Longa has been utilized in traditional medicine. Besides, it has been observed that curcumin as a strong antibacterial drug is authenticated to inhibit the growth of several bacteria including Staphylococcus aureus and Pseudomonas aeruginosa is well-known as it a good candidate for treating inflammatory diseases $(1,2)$ The ability of this drug in skin wound healing has been demonstrated on the other hand. In order to enhance wound healing, curcumin can increase biosynthesis of extracellular matrix proteins such as collagen $(3,4)$.

Chitosan is a linear polysaccharide derived from crustacean shells and fungi cell walls. It has been used as a biocompatible and safe material in drug delivery systems (5-7). It has been recently used in bandages and other haemostatic agents $(8,9)$. In addition, chitosan due to the ability of preventing the wound from being infected and dehydrated can optimize suitable conditions for healing (10). It has also been utilized as an antimicrobial agent that prevents the spread of infections into surrounding area $(11,12)$. Many studies have 
described the role of chitosan as a wound-healing accelerator. Chitosan could accelerate coagulation and enhance the functions of inflammatory cells (13-16). Moreover, it has been reported that chitosan could increase the tensile strength of wounds (17).

Nanoparticles are made from different biodegradable materials and their dimensions are generally less than $500 \mathrm{~nm}(18,19)$. Chitosan nanoparticles may be more efficient than chitosan solution at enhancing drug activity (20-23). Thus, chitosan-tripolyphosphate (TPP)-nanoparticles have been widely applied to deliver drugs across tissues. Overall, using chitosan-TPP nanoparticles as a nano-system can increase curcumin delivery in infectious tissue.

$(18,24,25)$.

\section{Objectives}

In the present study, we optimized an optimal method for curcumin loading in chitosan-TPP nanoparticles and investigated the antibacterial activity of curcumin-loaded chitosan-TPP nanoparticles on Staphylococcus aureus and Pseudomonas aeruginosa infections.

\section{Materials and Methods}

\subsection{Animals}

Female BALB/c mice were provided from Pasteur Institute of Iran (Iran, Tehran). All experiments were performed on 6 to 8 week-old mice in accordance with the guidelines of the Medical Ethics Committee of Baqiyatallah University of Medical Sciences (BMSU).

\subsection{Preparation of Bacterial Suspension}

Staphylococcus aureus (ATCC: 25923) and Pseudomonas aeruginosa (ATCC: 27853) were grown for $18 \mathrm{~h}$ in luria broth (LB) medium. The bacterial cells were finally collected, washed, and resuspended in sterile phosphate-buffered saline (PBS, pH 7.4). They were adjusted to a cell suspension of $10^{7}$ colony forming unit $(\mathrm{CFU}) / \mathrm{mL}$ using a UV spectrophotometer (Biophotometer, Eppendorf, Germany) in $620 \mathrm{~nm}$ wavelength.

\subsection{Curcumin-loaded chitosan-TPP nanoparti- cles synthesis}

Preparation of nanoparticles by ionotropic gelation method is based on electrostatic interac- tion between negatively- and positively-charged molecules such as poly anionic and cationic polymers. In the case of curcumin-loaded chitosanTPP nanoparticles, the amino groups existed on chitosan interacts with anionic groups of TPP salt. Stock solution of chitosan was made at $1 \mathrm{mg} / \mathrm{mL}$ in acidified distilled water (DW) and TPP was made at $1 \mathrm{mg} / \mathrm{mL}$ in DW. First, the chitosan stock solution $(1 \mathrm{~mL})$ was stirred for $10 \mathrm{~min}$ and its volume got adjusted to $1.5 \mathrm{~mL}$ with DW. Next, we added $5 \mu \mathrm{L}$ tween 80 to curcumin stock (1 $\mathrm{mg} / \mathrm{mL}$ ) solved in ethanol. Then, curcumin was added to the chitosan solution. Finally, TPP solution as a cross linker $(100 \mu \mathrm{L})$ were added to emulsified-curcumin-chitosan solution in a dropwise manner. The obtained solution was stirred for $30 \mathrm{~min}$ and centrifuged at $4000 \mathrm{~g}$ for $5 \mathrm{~min}$. At last, the supernatant was transferred into a new tube and kept for subsequent analysis.

\subsection{Curcumin-loaded chitosan-TPP nanoparti- cles characterization}

The samples were sonicated for $5 \mathrm{~min}$ in bath Wisd, WUC-D10H sonicator (Dihan, South Korea) before being analyzed and they were immediately used for measurements. The size and zeta potential of prepared nanoparticles were characterized by photon correlation spectroscopy (PCS) using a Malvern Zetasizer ZS series and Scattering Particle Size Analyzer (Malvern Co, UK). Following that, the shape, size and aggregation phenomena of curcumin-loaded chitosanTPP nanoparticles were measured by atomic force microscopy (AFM) (NVB-100, Olympus, Japan) and transmission electron microscopy (TEM) (Zeiss EM900, Carl Zeiss AG, Germany). Next, the fourier transforms infrared (FTIR) spectra of curcumin, chitosan and curcumin-loaded chitosan-TPP nanoparticles were assessed using Nicolet IR100 FTIR Spectrometer (Thermo, USA). Ultimately, the samples were mixed with pure potassium bromide $(\mathrm{KBr})$ as the background and compressed into discs using a manual tablet press.

\subsection{Evaluation of curcumin-loaded chitosan-TPP nanoparticles encapsulation}

To obtain nano-system with a maximum ratio of drug loading, different weight/weight ratios of chitosan/curcumin were tested. Therefore various amounts of curcumin were dissolved in a certain 
amount of chitosan-TPP nanoparticles. Subsequently the product was centrifuged at $20000 \mathrm{rpm}$ for $25 \mathrm{~min}$ and the supernatant of centrifuged curcumin-loaded chitosan-TPP nanoparticles formulation was checked for absorbance spectra by a spectrophotometer (Amersham Biosciences, Uppsala, Sweden) at $432 \mathrm{~nm}$. The loading efficiency was calculated using the following equation:

Encapsulation efficiency $(\%)=[($ Total amount of curcumin-Nonencapsulated curcumin) / Total amount of curcumin] $\times 100 \%$

\subsection{Determination of curcumin release profile from nanoparticles}

The release of curcumin from curcumin-loaded chitosan-TPP nanoparticles was evaluated using phosphate buffer $(\mathrm{pH}$ 7.4) and citrate buffer $(\mathrm{pH}$ 5.4) at $37^{\circ} \mathrm{C} .1 \mathrm{~mL}$ of the solution was poured into a dialysis bag (Spectrapor, MW cutoff 3500 $\mathrm{g} / \mathrm{mol}$ ) and placed into $100 \mathrm{~mL}$ of phosphate buffer ( $\mathrm{pH} 7.4)$ and citrate buffer $(\mathrm{pH} \mathrm{5.4)} \mathrm{sever-}$ ally. Afterward, $100 \mu \mathrm{L}$ tween 80 as an emulsifier agent was added in order to prevent the possible sedimentation of released drug. The release study was carried out at $37^{\circ} \mathrm{C}$ applying a shaking water bath (GFL, Burgwedel, Germany) and at the dedicated time intervals of $0,0.5,1,1.5,2,4,8$, $12,24,48,72$ and 96 hours, the sampling was performed. In each time point, $500 \mu \mathrm{L}$ of the sample was elicited, and then replaced by $500 \mu \mathrm{L}$ buffer. Then samples freeze dried and then dissolved in 2 $\mathrm{mL}$ of methanol. Finally all samples were examined by making use of spectrophotometer (Amersham Biosciences, Uppsala, Sweden) to determine the quantity of releasing curcumin. The accumulated release was calculated utilizing the following equation:

$$
\mathrm{R}=\left[\mathrm{V} \sum^{\mathrm{n}-\mathrm{i}}\left(\mathrm{C}_{\mathrm{i}}+\mathrm{V}_{0} \mathrm{C}\right)\right] / \mathrm{m}_{\mathrm{drug}}
$$

Where, $\mathrm{R}$ is the accumulated release $(\%), \mathrm{V}$ is the sampling volume, $\mathrm{V}_{0}$ is the initial volume, $\mathrm{C}_{\mathrm{i}}$ and $\mathrm{C}_{\mathrm{n}}$ are the curcumin concentrations, $\mathrm{i}$ and $\mathrm{n}$ are the sampling times, and $\mathrm{m}_{\mathrm{drug}}$ is the mass of curcumin in nanoparticles.

\subsection{Inoculation of bacterial infection on mouse skin}

$\mathrm{BALB} / \mathrm{c}$ mice were used in all these tests. The animals were anesthetized by ketamine and xylazine and inoculated with Staphylococcus aureus and Pseudomonas aeruginosa resuspended in PBS for $1 \mathrm{~h}$, with about $10^{7} \mathrm{CFU} /$ mouse/inoculation. The mice were divided into four groups. Each group was treated once a day with chitosanTPP nanoparticles, curcumin or curcumin-loaded chitosan-TPP nanoparticles for 3 days respectively. A non-treated group also used as a negative control. They were kept separately with free access to water and food. All the groups were sacrificed 4 days post infection, and the skin tissues were assessed for Staphylococcus aureus and Pseudomonas aeruginosa infection.

\subsection{Statistical Analysis}

Statistical analyses were performed using SPSS (Version 21, IBM, USA) software and graphical representations were showed by Excel 2007 (Version 12, Microsoft office, USA) software. Mann-Whitney U test was used to compare different groups and $p$ values less than 0.05 were considered statistically significant. The data are presented here as mean \pm SD of three independent experiments.

\section{Results}

\subsection{Curcumin-loaded chitosan-TPP nanoparti- cles properties}

Curcumin-loaded chitosan-TPP nanoparticles were prepared and optimized in size, charge and shape. The average diameter of nanoparticles was $160 \pm 10 \mathrm{~nm}$ and their surface charge was $+7 \pm 2$ $\mathrm{mV}$ (Figure 1). Curcumin-loaded chitosan-TPP nanoparticles showed the typical spherical shape (Figures 2 A-B). FTIR spectra of chitosan, curcumin and curcumin-loaded chitosan-TPP nanoparticles are shown in Figure 3. According to the Figure, two characterization peaks $\left(1103 \mathrm{~cm}^{-1}\right.$ of

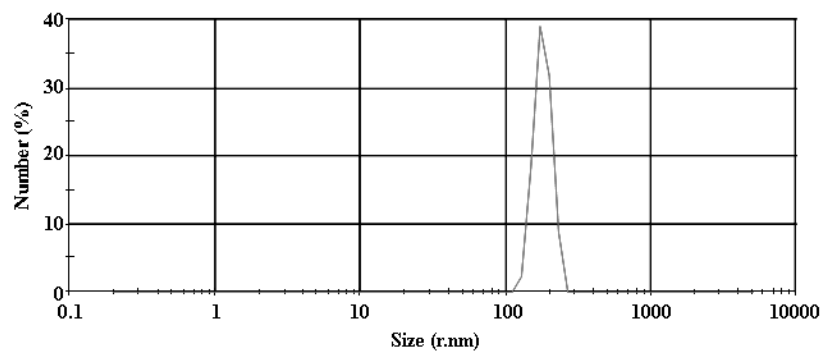

Figure 1. The average diameter of curcumin-loaded chitosan-TPP nanoparticles. The average diameter of these nanoparticles is $160 \pm 10 \mathrm{~nm}$ 
A

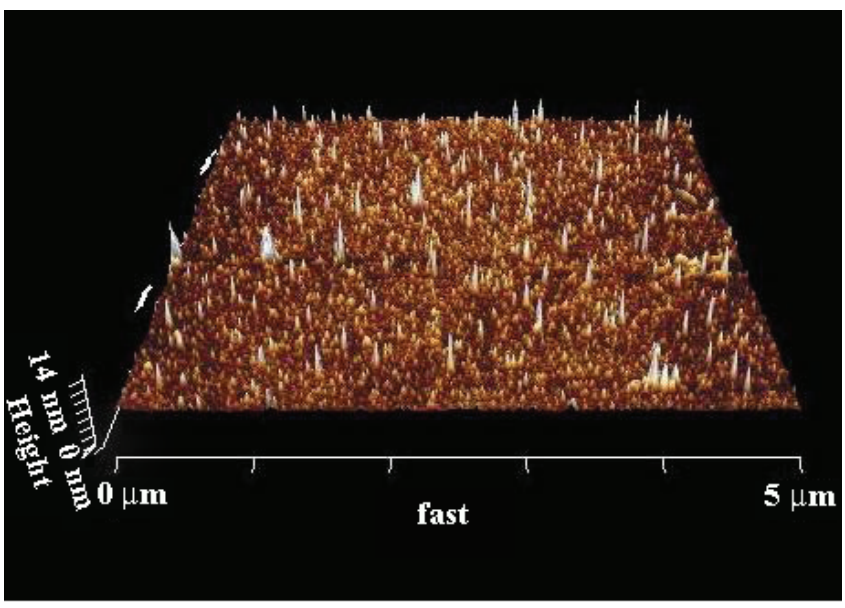

B

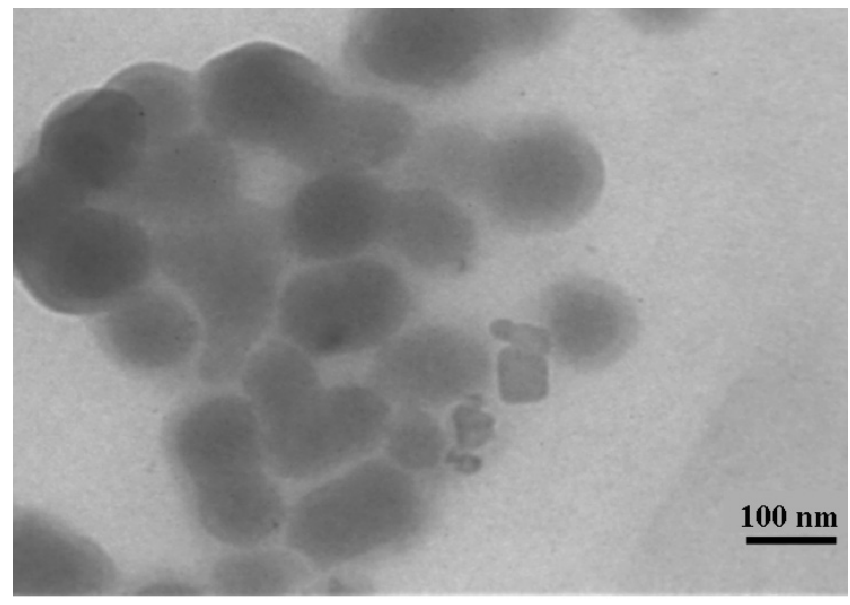

Figure 2. AFM (A) and TEM (B) of curcumin-loaded chitosan-TPP nanoparticles. The average diameter of these nanoparticles is $160 \pm 10 \mathrm{~nm}$ and their shape is typically spherical

$v(\mathrm{C}-\mathrm{O}-\mathrm{C})$ and $1419 \mathrm{~cm}^{-1}$ of $\left.v(\mathrm{OH})\right)$ existed in the spectrum of curcumin and three characterization peaks $\left(1033 \mathrm{~cm}^{-1}\right.$ of $v(\mathrm{C}-\mathrm{O}-\mathrm{C}), 1558 \mathrm{~cm}^{-1}$ and $1380 \mathrm{~cm}^{-1}$ of $\left.v\left(\mathrm{NH}_{2}\right)\right)$ existed in the spectrum of chitosan. In comparison with curcumin, a different spectrum was observed for curcuminloaded chitosan-TPP nanoparticles and new sharp peaks appeared at $2846 \mathrm{~cm}^{-1}$ and $1079 \mathrm{~cm}^{-1}$. Also, the $1419 \mathrm{~cm}^{-1}$ peak vibration shifted to $1689 \mathrm{~cm}^{-}$ 1. It can be supposed that the ammonium groups of chitosan were linked with hydroxide groups of curcumin in nanoparticles. The same results had been reported by the previous studies on curcumin loading in chitosan nanoparticles $(26,27)$.

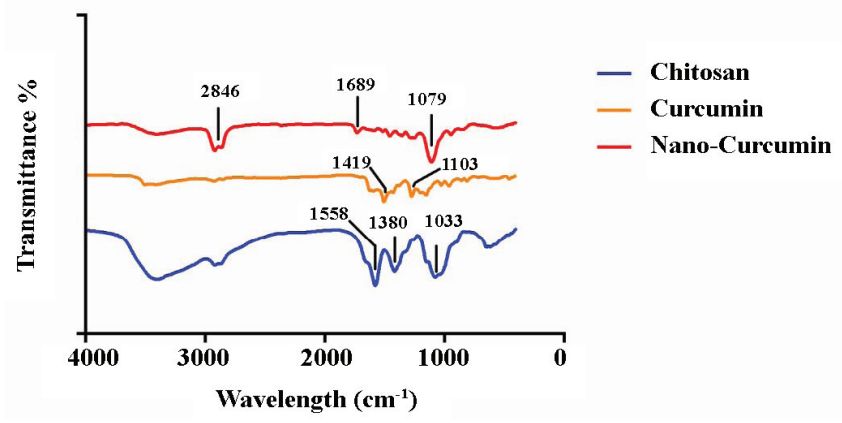

Figure 3. FTIR analysis of curcumin-loaded chitosan-TPP nanoparticles (Nano-curcumin). In comparison with curcumin, a different spectrum was observed for curcuminloaded chitosan-TPP nanoparticles and new sharp peaks appeared at $2846 \mathrm{~cm}^{-1}$ and $1079 \mathrm{~cm}^{-1}$. Also, the $1419 \mathrm{~cm}^{-1}$ peak vibration shifted to $1689 \mathrm{~cm}^{-1}$. It could be offered that curcumin was linked with chitosan in nanoparticles
4.2. The encapsulation efficiency of curcuminloaded chitosan-TPP nanoparticles

After the preparation of curcumin-loaded chitosan-TPP nanoparticles, this nano-formulation were centrifuged and harvested. The amount of curcumin remaining in the supernatant of the solution was then measured by a spectrophotometer. The encapsulation efficiency was determined $75 \pm$ $2 \%$ (Table 1 ).

\subsection{The profile of curcumin release from curcum- in-loaded chitosan-TPP nanoparticles}

According to the release curves, curcumin released from chitosan-TPP nanoparticles over a $96 \mathrm{~h}$ period and this time was slower at $\mathrm{pH} 7.4$ as compared to $\mathrm{pH}$ 5.4. In comparison with the release profiles of free curcumin, there are similar release profiles at $\mathrm{pH} 7.4$ and 5.4. Under the studied conditions, within $48 \mathrm{~h}, 41.19 \%$ and $82.26 \%$ of physically loaded curcumin was released at $\mathrm{pH}$ 7.4 and 5.4 , respectively and during $72 \mathrm{~h}, 42.23 \%$ and $93.75 \%$ of physically loaded curcumin was released at $\mathrm{pH} 7.4$ and 5.4, respectively. After 96 h, $52.41 \%$ and $98.91 \%$ of physically loaded curcumin was released at $\mathrm{pH} 7.4$ and 5.4, in order (Figure 4).

\subsection{The inhibition of bacterial infection by cur-} cumin-loaded chitosan-TPP nanoparticles

The infected mice were treated with curcuminloaded chitosan-TPP nanoparticles in comparison with the non-treated ones and evaluated for the 
Table 1. The encapsulating efficiency (\%) of curcumin loaded chitosan-TPP nanoparticles. The most encapsulation efficiency was determined $75 \pm 2 \%$

\begin{tabular}{lcc}
\hline $\begin{array}{l}\text { Chitosan concen- } \\
\text { tration } \mathbf{~ m g / m L}\end{array}$ & $\begin{array}{c}\text { Curcumin concen- } \\
\text { tration } \mathbf{~ m g / m L}\end{array}$ & $\begin{array}{c}\text { Encapsulation } \\
\text { efficiency (\%) }\end{array}$ \\
\hline 1 & 0.75 & $59 \pm 1.6$ \\
1 & 1 & $75 \pm 2$ \\
1 & 1.25 & $71 \pm 2.6$ \\
1 & 1.5 & $67 \pm 1.3$ \\
1 & 1.75 & $63 \pm 1.5$ \\
1 & 2 & $60 \pm 1.5$ \\
\hline
\end{tabular}

expression of bacterial count. The count of Staphylococcus aureus was analyzed to explore the effect of curcumin, chitosan-TPP nanoparticles and curcumin-loaded chitosan-TPP nanoparticles on infection. The statistical analyses showed that curcumin-loaded chitosan-TPP nanoparticles significantly decreased the growth of Staphylococcus aureus (Mann-Whitney U test; $p<0.05 \%$ ), compared to the control groups (Figure 5). The count of Pseudomonas aeruginosa was analyzed to explore the effect of curcumin, chitosan-TPP nanoparticles and curcumin-loaded chitosan-TPP nanoparticles on infection. The statistical analyses showed that curcumin-loaded chitosan-TPP nanoparticles significantly decreased the growth of Pseudomonas aeruginosa (Mann-Whitney $\mathrm{U}$ test; $p<0.05 \%$ ), compared to the control groups (Figure 6).

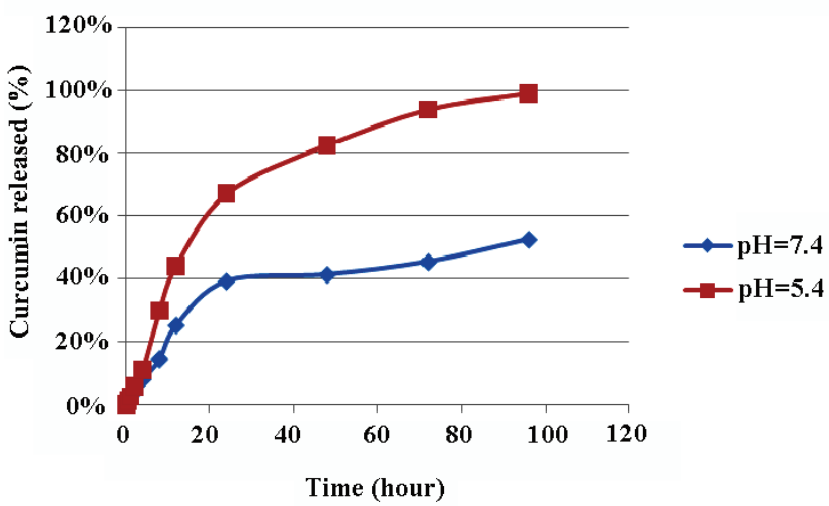

Figure 4. Release curves of curcumin-loaded chitosan-TPP nanoparticles. According to the release curves, curcumin released from chitosan-TPP nanoparticles over a $96 \mathrm{~h}$ period and this time was slower at $\mathrm{pH} 7.4$ as compared to $\mathrm{pH} 5.4$

\section{Discussion}

Curcumin as a potent, biocompatible and bioactive agent with an antimicrobial property has been applied in nanoparticles synthesis $(28,29)$. In the present study, curcumin was loaded in chitosan-TPP nanoparticles by ionotropic gelation method. AFM and TEM revealed the average diameter of $160 \pm 10 \mathrm{~nm}$ and a spherical shape for curcumin-loaded chitosan-TPP nanoparticles. By contrast, Das et.al loaded curcumin in chitosan nanoparticles by applying sodium alginate as a cross linker instead of TPP salt. Their nanoparticles size was $100 \pm 20 \mathrm{~nm}$ with spherical shape (27). Akhtar et.al also produced curcumin-loaded chitosan nanoparticles with size of more than 200 $\mathrm{nm}$ while curcumin-loaded chitosan nanoparticles with size of $160 \mathrm{~nm}$ were made in our research (30). Here, surface charge of the produced curcumin-loaded chitosan nanoparticles was $+7 \pm 2$ $\mathrm{mV}$ whereas the surface charge of chitosan-TPP nanoparticles alone has been reported $+25 \pm 4 \mathrm{mV}$. As a result, by loading curcumin the charge of chitosan-TPP nanoparticles decreased (26). After curcumin loading in nanoparticles, curcuminloaded chitosan nanoparticles got soluble and a transparent solution was obtained. The transparency of nanoparticles solution indicated that curcumin as a hydrophobic material can be emulsified in chitosan-TPP nanoparticles along with applying tween 80 emulsifier. In the present study,

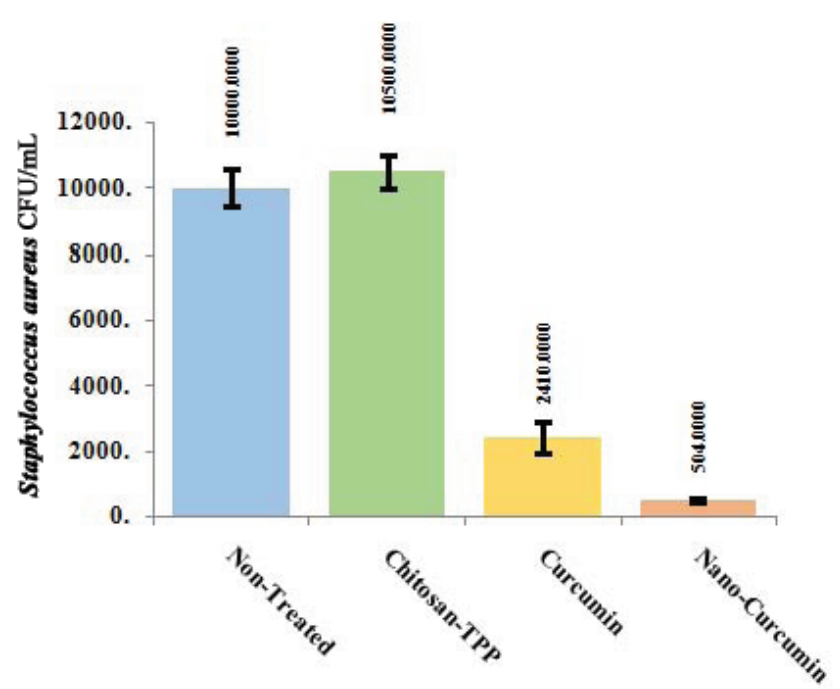

Figure 5. Antibacterial activity of curcumin-loaded chitosan-TPP nanoparticles (Nano-curcumin). Curcuminloaded chitosan-TPP nanoparticles significantly inhibited Staphylococcus aureus infection (Mann-Whitney U test; $p<0.05 \%$ ), compared to chitosan-TPP and curcumin 


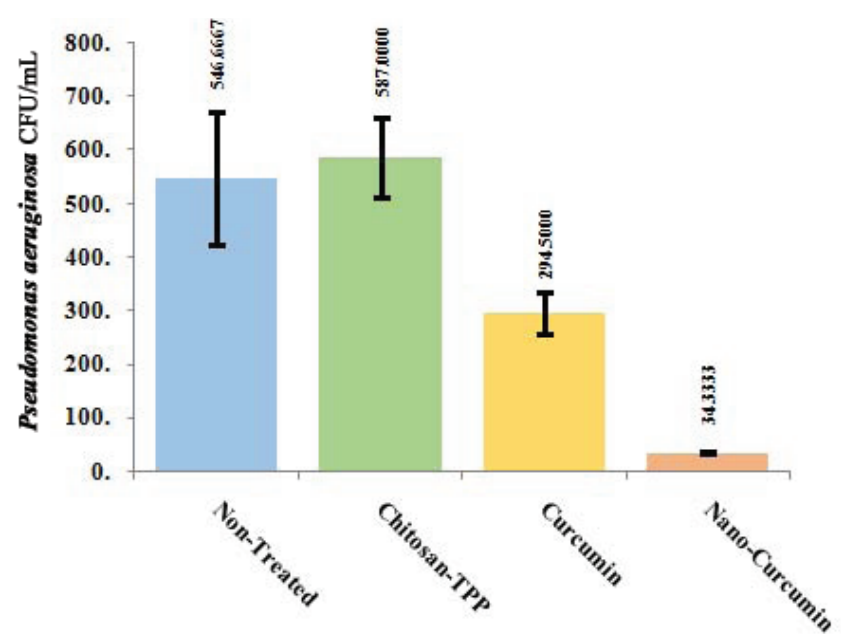

Figure 6. Antibacterial activity of curcumin-loaded chitosan-TPP nanoparticles (Nano-curcumin). Curcuminloaded chitosan-TPP nanoparticles significantly inhibited Pseudomonas aeruginosa infection (Mann-Whitney U test; $p<0.05 \%$ ), compared to the chitosan-TPP and curcumin

the encapsulation efficiency of curcumin in chitosan-TPP nanoparticles was $75 \pm 2 \%$ while in Das et al., study the entrapment efficiency was less than 20\% (27). Probably, using TPP cross linker caused to increase of curcumin loading in chitosan-TPP nanoparticles. The peaks characterized by FTIR analysis indicated that chitosan was linked to TPP salt by ammonium groups of chitosan and phosphate groups of TPP (26). Curcumin is more likely to link to chitosan via involving the hydroxide groups of curcumin and ammonium groups of chitosan (27).

To compare with Das et al., study in which the kinetic of release showed $80 \%$ release of curcumin from nanoparticles, in our survey $52.41 \%$ and $98.91 \%$ of physically loaded curcumin was released at $\mathrm{pH} 7.4$ and 5.4 within $96 \mathrm{~h}$, respectively. The slower release of curcumin from curcumin-loaded chitosan-TPP nanoparticles at $\mathrm{pH} 7.4$ is perhaps due to chitosan nature as this molecule is more soluble and degradable in acidic $\mathrm{pH}$ than neutral and/or alkaline $\mathrm{pH}$ (27). For the mentioned results, we used curcumin-loaded chitosan-TPP nanoparticles to suppress both Gram negative and Gram positive bacteria including Staphylococcus aureus and Pseudomonas aeruginosa. This study has shown that curcumin-loaded chitosan-TPP nanoparticles could significantly suppress the progression of Staphylococcus aureus and Pseudomonas aeruginosa infection on mouse skin, whereas chitosan-TPP by itself could not inhibit bacterial infection. We observed that antimicrobial effect of curcumin-loaded chitosanTPP nanoparticles is significantly better than individual curcumin. Nanoparticles in drug delivery systems are able to diminish the defects of usual delivery systems $(18,25)$ and it is probably due to the gradual release of curcumin by nanoparticles in infection areas. Accordingly, combination of chitosan-TPP nanoparticles and curcumin produces a synergy effect on the antibacterial activity that is more than both of them individually. Bhawana et al. have also displayed that curcumin was able to inhibit the bacterial infection. They demonstrated that the cytotoxicity effect of curcumin-loaded chitosan-TPP nanoparticles was greater on Gram-positive bacteria than Gram-negative bacteria (29). Our experiments showed the efficacy and effectiveness of curcumin-loaded chitosan-TPP nanoparticles on both Gram-positive and Gram-negative bacteria including Staphylococcus aureus and Pseudomonas aeruginosa. De et al. have also elucidated antimicrobial activity of curcumin against helicobacter pylori (28). According to Bansal et al., research, curcumin was able to inhibit nosocomial infections caused by Klebsiella pneumonia, an opportunistic pathogen (31). These studies have been shown antimicrobial effect of curcumin while our study has displayed a higher potency of curcuminloaded chitosan-TPP nanoparticles rather than the individual curcumin. In another research, Singh et al., studied antibacterial properties of curcumin. They declared that curcumin was a potent molecule in the treatment of bacterial infections (1). In agreement with our study, Rai et al., suggested that curcumin might be considered as an important antibacterial drug target. In this study, curcumin has been shown to have a potent antibacterial activity against a number of pathogenic bacteria including Staphylococcus aureus, Staphylococcus epidermidis and Enterococcus (32). Also, Wang et al. used microcapsule curcumin against Staphylococcus aureus, Escherichia coli, Yersinia enterocolitica, Bacillus subtilis and Bacillus cereus. Therefore, their observation indicated that curcumin induced a spectrum inhibitory effect against all these organisms (2).

To conclude, curcumin-loaded chitosan-TPP nanoparticles can potentially be utilized in drug delivery systems and applied as a strategy to specifically activate antibacterial system at the 
same time. This property of curcumin-loaded chitosan-TPP nanoparticles introduces them as a good candidate for drug targeting of bacterial infection including Staphylococcus aureus and Pseudomonas aeruginosa infection.

\section{Acknowledgments}

This study was funded by a dissertation grant from the Molecular Biology Research Center, Baqiyatallah University of Medical Sciences (BMSU).

\section{References}

1. Singh RK, Rai D, Yadav D, Bhargava A, Balzarini J, De Clercq E. Synthesis, antibacterial and antiviral properties of curcumin bioconjugates bearing dipeptide, fatty acids and folic acid. Eur J Med Chem.2010;45(3):1078-86. doi:10.1016/j.ejmech.2009.12.002

2. Wang Y, Lu Z, Wu H, Lv F. Study on the antibiotic activity of microcapsule curcumin against foodborne pathogens. Int J Food Microbiol. 2009;136(1):71-4. doi:10.1016/j.ijfoodmicro.2009.09.001

3. Panchatcharam M, Miriyala S, Gayathri VS, Suguna L. Curcumin improves wound healing by modulating collagen and decreasing reactive oxygen species. Mol Cell Biochem. 2006;290(1-2):87-96. doi:10.1007/s11010-006-9170-2

4. Sidhu GS, Mani H, Gaddipati JP, Singh AK, Seth P, Banaudha KK, Patnaik GK, Maheshwari RK. Curcumin enhances wound healing in streptozotocin induced diabetic rats and genetically diabetic mice. Wound Repair Regen. 1999;7(5):362-74. doi: 10.1046/j.1524-475X.1999.00362.x

5. Correlo VM, Boesel LF, Bhattacharya M, Mano JF, Neves NM, Reis RL. Hydroxyapatite Reinforced Chitosan and Polyester Blends for Biomedical Applications. Macromol Mater Eng. 2005;290(12):1157-65.doi: 10.1002/mame.200500163

6. De Jong WH, Borm PJ. Drug delivery and nanoparticles: applications and hazards. Int J Nanomedicine. 2008;3(2):133-49. doi: 10.2147/IJN.S596

7. Di Martino A, Sittinger M, Risbud MV. Chitosan: a versatile biopolymer for orthopaedic tissue-engineering. Biomaterials 2005;26(30):5983-90. doi:10.1016/j.biomaterials.2005.03.016

8. Kozen BG, Kircher SJ, Henao J, Godinez FS, Johnson AS. An alternative hemostatic dressing: comparison of CELOX, HemCon, and QuikClot. Academic emergency medicine: Official $J$ Acad Emerg Med. 2008;15(1):7481.doi:10.1111/j.1553- 2712.2007.00009.x

9. Millner RW, Lockhart AS, Bird H, Alexiou C. A new hemostatic agent: initial life-saving experience with Celox (chitosan) in cardiothoracic surgery. Ann Thorac Surg.2009;87(2):e13-4. doi:http://dx.doi.org/10.1016/j.athoracsur.2008.09.046

10. Ueno H, Mori T, Fujinaga T. Topical formulations and wound healing applications of chitosan. Adv Drug Deliv Rev. 2001;52(2):105-15. doi:10.1016/S0169-409X(01)00189-2
11. Robson MC. Wound infection. A failure of wound healing caused by an imbalance of bacteria. Surg Clin North Am. 1997;77(3):637-50.

12. Dai T, Tanaka M, Huang YY, Hamblin MR. Chitosan preparations for wounds and burns: antimicrobial and wound-healing effects. Expert Rev Anti Infect Ther. 2011;9(7):85779.doi:10.1586/eri.11.59

13. Santos TC, Marques AP, Silva SS, Oliveira JM, Mano JF, Castro AG, Castro AG, Reis RL. In vitro evaluation of the behaviour of human polymorphonuclear neutrophils in direct contact with chitosan-based membranes. J Biotechnol. 2007;132(2):218-26.doi:10.1016/j.jbiotec.2007.07.497

14. Ueno H, Yamada H, Tanaka I, Kaba N, Matsuura M, Okumura M, Okumura M, Okumura M, Kadosawa T, Fujinaga T. Accelerating effects of chitosan for healing at early phase of experimental open wound in dogs. Biomaterials 1999;20(15):1407-14.doi:10.1016/S0142-9612(99)00046-0

15. Peluso G, Petillo O, Ranieri M, Santin M, Ambrosio L, Calabro D,Avallone B, Balsamo G. Chitosan-mediated stimulation of macrophage function. Biomaterials 1994;15(15):121520.doi:10.1016/0142-9612(94)90272-0

16. Nascimento EG, Sampaio TB, Medeiros AC, Azevedo EP. Evaluation of chitosan gel with $1 \%$ silver sulfadiazine as an alternative for burn wound treatment in rats. Acta Cirurgica Brasileira. 2009;24(6):460-5.http://dx.doi.org/10.1590/S010286502009000600007

17. Degim Z, Celebi N, Sayan H, Babul A, Erdogan D, Take G. An investigation on skin wound healing in mice with a taurine-chitosan gel formulation. Amino Acids. 2002;22(2):18798.doi:10.1007/s007260200007

18. Anitha A, Maya S, Deepa N, Chennazhi KP, Nair SV, Tamura H, Tamura H, Jayakumar R. Efficient water soluble O-carboxymethyl chitosan nanocarrier for the delivery of curcumin to cancer cells. Carbohydrate Polymers. 2011;83(2):45261.doi:10.1016/j.carbpol.2010.08.008

19. Eidi H, Joubert O, Némos C, Grandemange S, Mograbi B, Foliguet B, Toumebize J, Maincent P, LeFaou A.Aboukhamis I, Rihn BH. Drug delivery by polymeric nanoparticles induces autophagy in macrophages. Int $J$ Pharm.2012;422(1-2):495503.doi:10.1016/j.jpharm.2011.11.020

20. Fernandez-Urrusuno R, Calvo P, Remunan-Lopez C, Vila-Jato JL, Alonso MJ. Enhancement of nasal absorption of insulin using chitosan nanoparticles. Pharm Res. 1999;16(10):157681.10.1023/A:1018908705446

21. Ma Z, Lim LY. Uptake of chitosan and associated insulin in Caco-2 cell monolayers: a comparison between chitosan molecules and chitosan nanoparticles. Pharm Res. 2003;20(11):18129.doi:10.1023/B:PHAM.0000003379.76417.3e

22. Ma Z, Lim TM, Lim L-Y. Pharmacological activity of peroral chitosan-insulin nanoparticles in diabetic rats. Int J Pharm. 2005;293(1-2):271-80.doi:10.1016/j.ijpharm.2004.12.025

23. Pan Y, Li YJ, Zhao HY, Zheng JM, Xu H, Wei G, Hao JS, Cui FD. Bioadhesive polysaccharide in protein delivery system: chitosan nanoparticles improve the intestinal absorption of insulin in vivo. Int $J$ Pharm. 2002;249(1-2):13947.doi:10.1016/S0378-5173(02)00486-6

24. Liu Z, Jiao Y, Wang Y, Zhou C, Zhang Z. Polysaccharides- 
based nanoparticles as drug delivery systems. Adv Drug Deliv Rev. 2008;60(15):1650-62.doi:10.1016/j.addr.2008.09.001

25. Lu B, Liu X, Huang Z, Xu H, Xu P, Wang Y, Zheng H, Yin Y, Zhang X, Zhuo R. Synthesis of diamine maleyl chitosans, and in vitro transfection studies. Carbohydr Polym. 2012;87(2):1453-1459.doi:10.1016/j.carbpol.2011.09.039

26. Mofazzal Jahromi M, Karimi M, Azadmanesh K, Naderi Manesh H, Hassan Z, Moazzeni S. The effect of chitosantripolyphosphate nanoparticles on maturation and function of dendritic cells. Comp Clin Pathol. 2014;23:14211427.doi:10.1007/s00580-013-1799-0

27. Das RK, Kasoju N, Bora U. Encapsulation of curcumin in alginate-chitosan-pluronic composite nanoparticles for delivery to cancer cells. Nanomedicine 2010;6(1):153160.doi:http://dx.doi.org/10.1016/j.nano.2009.05.009

28. De R, Kundu P, Swarnakar S, Ramamurthy T, Chowdhury A, Nair GB, Mukhopadhyay AK. Antimicrobial activity of curcumin against Helicobacter pylori isolates from India and during infections in mice. Antimicrob Agents chemother.2009;53(4):1592-1597.doi: 10.1128/AAC.01242-08

29. Bhawana, Basniwal RK, Buttar HS, Jain VK, Jain N. Curcumin nanoparticles: preparation, characterization, and antimicrobial study. J Agric Food Chem. 2011;59(5):205661.doi:10.1021/ff104402t

30. Akhtar F, Rizvi MM, Kar SK. Oral delivery of curcumin bound to chitosan nanoparticles cured Plasmodium yoelii infected mice. Biotechnol Adv. 2012;30(1):310320.doi:10.1016/j.biotechadv.2011.05.009

31. Bansal S, Chhibber S. Curcumin alone and in combination with augmentin protects against pulmonary inflammation and acute lung injury generated during Klebsiella pneumoniae B5055induced lung infection in BALB/c mice. $J$ Med Microbiol. 2010;59(Pt 4):429-37.doi: 10.1099/jmm.0.016873-0

32. Rai D, Singh JK, Roy N, Panda D. Curcumin inhibits FtsZ assembly: an attractive mechanism for its antibacterial activity. Biochem J. 2008;410(1):147-55.doi:10.1042/BJ20070891 